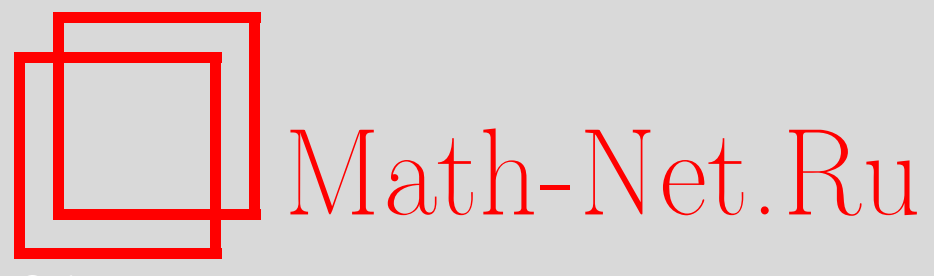

К. И. Облаков, Т. А. Облакова, Вложения графов в евклидово пространство, при которых число точек, принадлежащих гиперплоскости, минимально, Матем. сб., 2012, том 203, номер 10, 145-160

DOI: https://doi.org/10.4213/sm7878

Использование Общероссийского математического портала Math-Net.Ru подразумевает, что вы прочитали и согласны с пользовательским соглашением http://www . mathnet.ru/rus/agreement

Параметры загрузки:

IP : 52.6 .47 .48

26 апреля 2023 г., 13:50:24

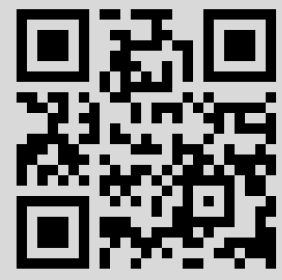




\author{
К. И. Облаков, Т. А. Облакова
}

\title{
Вложения графов в евклидово пространство, при которых число точек, принадлежащих гиперплоскости, минимально
}

\begin{abstract}
Работа посвящена такой характеристике графа, как минимальное по всем вложениям графа в пространство данной размерности число точек, лежащих в одной гиперплоскости. Даются верхняя и нижняя оценки этого числа, линейно зависящие от размерности пространства. Для деревьев получена более точная верхняя оценка, асимптотически совпадающая с нижней при большой размерности пространства.

Библиография: 9 названий.
\end{abstract}

Ключевые слова: граф, вложение, гиперплоскость.

\section{$\S 1$. Введение}

Настоящая работа продолжает исследование, начатое авторами в [1] и [2], рассматриваются специальные вложения графов в евклидово пространство. Различные вложения графов рассматривались в математике в течение последнего полувека. Классическими стали теоремы о вложении произвольного графа в трехмерное пространство, о минимальности и полноте семейства графов Куратовского с точки зрения невложимости в плоскость.

К. Борсуком было введено понятие $k$-регулярных вложений компактов [3]. В. Г. Болтянский, С. С. Рышков и Ю.А. Шашкин исследовали их для случая полиэдров [4]. Также ими занимались С. А. Богатый и В. М. Вылов [5], [6]. На вложения, рассматриваемые в этой работе, накладываются более слабые и в некотором смысле более общие условия чем $k$-регулярность - ограничивается количество точек, которые может содержать произвольная гиперплоскость. Практически единственным результатом, касающимся этого семейства вложений, является теорема Мэрхьюбера о том, что любое компактное множество в $n$-мерном пространстве, содержащее не более $n$ точек на любой гиперплоскости, является гомеоморфным образом замкнутой части окружности [7].

\section{§ 2. Основные определения и свойства}

Везде через $n$ будем обозначать размерность пространства. Максимальное по всем гиперплоскостям число точек некоторого множества на гиперплоскости назовем числом гиперпланарности данного множества. Так как любые $n$ точек задают не меньше одной гиперплоскости, понятно, что число гиперпланарности любого множества, содержащего по крайней мере $n$ точек, не может быть меньше $n$. 
Числом $n$-гиперпланарности графа будем называть минимум по всем вложениям данного графа в $n$-мерное пространство числа гиперпланарности образа. Если размерность пространства ясна из контекста, это число называется просто числом гиперпланарности графа. Для оценки сверху числа гиперпланарности построим вложения с малым числом гиперпланарности.

Основной конструкцией для анализа вложений с малым числом гиперпланарности является моментная кривая $\left(t, t^{2}, t^{3}, \ldots, t^{n}\right)$, рассматриваемая в теории многогранников [8]. Она обладает очень важным свойством с точки зрения рассматриваемых вложений - любая гиперплоскость пересекает ее не более чем по $n$ точкам.

Полиномиальной кривой будем называть афинный образ моментной кривой. Так как афинные преобразования не меняют число гиперпланарности, то верно следующее

ПРЕДЛОЖЕНИЕ 1. Число гиперпланарности полиномиальной кривой равно $n$.

Также будет использоваться следующее

ПреДЛОжЕНИЕ 2. Две различные полиномиалъные кривые могут иметь не более чем $n^{2}$ точек пересечения.

ДоказАтельство. Рассмотрим две различные полиномиальные кривые $l(t)$ и $s(t)$. Пусть $O_{1}, O_{2}, \ldots, O_{m}$ - их точки пересечения. Пусть $\left\{x_{1}, x_{2}, \ldots, x_{n}\right\}-$ система координат, в которой кривая $l(t)$ является моментной, а $\left\{y_{1}, y_{2}, \ldots, y_{n}\right\}-$ система координат, в которой кривая $s(t)$ является моментной.

Рассмотрим случай, когда оси $x_{1}$ и $y_{1}$ параллельны. В таком случае можно обе кривые привести к параметру $x_{1}$. Получим: $l\left(x_{1}\right)=\left\{x_{1}, x_{1}^{2}, \ldots, x_{1}^{n}\right\}, s\left(x_{1}\right)=$ $\left\{x_{1}, P_{2}\left(x_{1}\right), \ldots, P_{n}\left(x_{1}\right)\right\}$, где $P_{i}$ - линейно независимые полиномы степени не выше $n$.

Заметим, что для каждой точки $O_{i}$ с координатами $\left(x_{1, i}, x_{2, i}, \ldots, x_{n, i}\right)$ верны равенства

$$
x_{1, i}^{j}=P_{j}\left(x_{1, i}\right) .
$$

Так как степень каждого $P_{j}$ не превышает $n$, при $m \geqslant n+1$ совпадение $x_{1, i}^{j}$ и $P_{j}\left(x_{1, i}\right)$ в $m$ точках $O_{i}$ означает, что $P_{j}=x_{1}^{j}$. Это противоречит тому, что кривые $l$ и $s$ различны. Значит, в рассматриваемом случае число точек пересечения кривых не может превышать $n$, и тем более $n^{2}$. Для случая параллельности осей $x_{1}$ и $y_{1}$ предложение доказано.

Пусть теперь оси $x_{1}$ и $y_{1}$ не параллельны. Выразим единичный вектор по оси $y_{1}$ через векторы системы $\left\{x_{i}\right\}$. Получим соотношение $y_{1}=a_{0}+a_{1} x_{1}+a_{2} x_{2}+$ $\cdots+a_{n} x_{n}$. Заметим, что для каждой точки на кривой $l$ верны соотношения $x_{2}=x_{1}^{2}, x_{3}=x_{1}^{3}, \ldots, x_{n}=x_{1}^{n}$. Значит, каждая точка $O_{i}$ удовлетворяет уравнению $y_{1}=a_{0}+a_{1} x_{1}+a_{2} x_{1}^{2}+\cdots+a_{n} x_{1}^{n}$. Так как оси не параллельны, это уравнение не является линейным. 
Проведем аналогичные рассуждения для кривой $s(t)$ и координат $y_{i}$. Получим $x_{1}=b_{0}+b_{1} y_{1}+b_{2} y_{2}+\cdots+b_{n} y_{n}$. Для каждой точки кривой $s(t)$, и в частности для точек $O_{i}$, получим соотношение $x_{1}=b_{0}+b_{1} y_{1}+b_{2} y_{1}^{2}+\cdots+b_{n} y_{1}^{n}$. Это уравнение также не является линейным.

Теперь подставим одно из полученных равенств для точек $O_{i}$ в другое. Получим, что для всех точек $O_{i}$ верно равенство

$$
\begin{aligned}
x_{1}=b_{0} & +b_{1}\left(a_{0}+a_{1} x_{1}+a_{2} x_{1}^{2}+\cdots+a_{n} x_{1}^{n}\right)+b_{2}\left(a_{0}+a_{1} x_{1}+a_{2} x_{1}^{2}+\cdots+a_{n} x_{1}^{n}\right)^{2} \\
& +\cdots+b_{n}\left(a_{0}+a_{1} x_{1}+a_{2} x_{1}^{2}+\cdots+a_{n} x_{1}^{n}\right)^{n} .
\end{aligned}
$$

Из нелинейности уравнений $y_{1}=a_{0}+a_{1} x_{1}+a_{2} x_{1}^{2}+\cdots+a_{n} x_{1}^{n}$ и $x_{1}=b_{0}+b_{1} y_{1}+$ $b_{2} y_{1}^{2}+\cdots+b_{n} y_{1}^{n}$ следует, что полученное уравнение не является тождеством. Перенеся $x_{1}$ в правую часть равенства, получим полином степени не более $n^{2}$. Ясно, что значений $x_{1}$, удовлетворяющих этому равенству, не более $n^{2}$. Так как различные точки кривой $l$ не могут иметь одинаковую координату $x_{1}$, из этого следует, что точек пересечения кривых не больше чем $n^{2}$. Предложение доказано.

\section{§ 3. Верхняя оценка в общем случае}

3.1. Обобщенная моментная кривая. Введем еще одно важное понятие. Пусть $M(t)=\left(t, t^{2}, \ldots, t^{n}\right)-$ моментная кривая, $\vec{v}(t)=\left(1,2 t, 3 t^{2}, \ldots, n t^{n-1}\right)-$ ее вектор скорости. Запишем очевидное равенство $M(t)=\int_{0}^{t} \vec{v}(\tau) d \tau$.

ОПРЕДЕЛЕНИЕ 1. Обобщенной моментной кривой назовем кривую $S(t)$, задаваемую уравнением

$$
S(t)=\vec{C}+\int_{a}^{t} \vec{v}(\tau) k(\tau) d \tau
$$

где $k(\tau)$ - бесконечно гладкая неотрицательная скалярная функция. Функция $k$ может обращаться в нуль на целых отрезках, в таком случае кривая при этих значениях $t$ вырождается в точку. В частности, при $k \equiv 0$ вся кривая вырождается в точку.

ЗАмечАниЕ 1. Выбор а влияет только на запись, но не на форму кривой. Так, если имеется обобщенная моментная кривая $S_{1}(t)=\vec{C}_{1}+\int_{a}^{t} \vec{v}(\tau) k(\tau) d \tau$, то для любого $b$ кривая $S_{2}(t)=\vec{C}_{2}+\int_{b}^{t} \vec{v}(\tau) k(\tau) d \tau$, где $\vec{C}_{2}=\vec{C}_{1}+\int_{a}^{b} \vec{v}(\tau) k(\tau) d \tau$, совпадает с $S_{1}$.

ПРЕДЛОЖЕНИЕ 3. Для любых попарно различных $\tau_{1}, \tau_{2}, \ldots, \tau_{n}$ векторы $v\left(\tau_{1}\right), v\left(\tau_{2}\right), \ldots, v\left(\tau_{n}\right)$ линейно независимъ.

ДокАЗАтельство. Вектор $v\left(t_{0}\right)$ имеет вид $\left(1,2 t_{0}, 3 t_{0}^{2}, \ldots, n t_{0}^{n-1}\right)$. Заметим, что определитель, составленный из $n$ векторов $v\left(\tau_{i}\right)$, является определителем Вандермонда, домноженным на коэффициент $n$ !, и потому не равен нулю. Это равносильно линейной независимости векторов. 
Докажем, что обобщенная моментная кривая обладает основным свойством моментной кривой.

ТЕОРема 1. Число гиперпланарности обобщенной моментной кривой равно $n$.

ДокАзАтЕльство. Пусть нашлись $n+1$ точка на кривой, лежащие в одной гиперплоскости $\alpha$. Интеграл $\int \vec{v}(\tau) k(\tau) d \tau$ на $n$ промежутках между ними равен вектору смещения вдоль плоскости $\alpha$ и, следовательно, параллелен $\alpha$. Из этого и из принципа непрерывности следует, что на каждом из $n$ промежутков есть значение $\vec{v}(\tau)$, параллельное $\alpha$. Но координаты $n$ векторов линейно независимы и не могут лежать в $(n-1)$-мерном пространстве векторов, параллельных $\alpha$. Полученное противоречие доказывает теорему.

3.2. Ветвящаяся моментная кривая. Введем конструкцию, позволяющую вкладывать графы в пространство с малым числом гиперпланарности образа.

ОПреДЕЛЕНИЕ 2. Набор обобщенных моментных кривых, получающихся за счет разных $k(\tau)$ и $\vec{C}$, назовем ветвящейся моментной кривой, а кривую из этого набора - ветвъю.

Из определения 2 и теоремы 1 следует

ПрЕДЛОЖЕНИЕ 4. Число гиперпланарности ветвящейся моментной кривой с $m$ ветвями не превосходит $\mathrm{nm}$.

ОПРЕДЕЛЕНИЕ 3. Рассмотрим некоторую обобщенную моментную кривую $S_{0}(t)=\vec{C}_{0}+\int_{0}^{t} \vec{v}(\tau) k_{0}(\tau) d \tau$. Если носитель некоторой бесконечно гладкой и неотрицательной функции $p(t)$ лежит на отрезке $\left[t_{1}, t_{2}\right]$ и обобщенная моментная кривая $S(t)$ определяется формулой $S(t)=\vec{C}_{0}+\int_{0}^{t} \vec{v}(\tau)\left(k_{0}(\tau)+p(\tau)\right) d \tau$, то $S(t)=S_{0}(t)+\int_{t_{1}}^{t_{2}} \vec{v}(\tau) p(\tau) d \tau$ при $t \geqslant t_{2}$, и $S(t)=S_{0}(t)$ при $t \leqslant t_{1}$. Вектор $\int_{t_{1}}^{t_{2}} \vec{v}(\tau) p(\tau) d \tau$ в этом случае будем называть добавочным вектором для функции $p$ и кривой $S(t)$.

Заметим, что добавочный вектор линейно зависит от функции $p$ из-за линейности интеграла.

\section{3. Теорема о реализации абстрактной схемы.}

ОПРЕДЕЛЕНИЕ 4. Назовем абстрактной схемой ветвления с $m$ ветвями набор $(T, m, A)$, где $T$ - конечное множество точек на прямой $t_{0}<t_{1}<\cdots<t_{q}$, $m$ - натуральное число, $A$ - набор условий одного из следующих видов, $j, k \leqslant m$ :

- "на интервале $\left(t_{i}, t_{i+1}\right)$ ветви с номерами $j$ и $k$ совпадают";

- "на интервале $\left(t_{i}, t_{i+1}\right)$ ветви с номерами $j$ и $k$ не совпадают";

- "в точке $t_{i}$ ветви с номерами $j$ и $k$ совпадают";

- "в точке $t_{i}$ ветви с номерами $j$ и $k$ не совпадают". 
На набор высказываний $A$ налагаются следующие условия:

- полнота: для каждой пары ветвей и для каждого интервала $\left(t_{i}, t_{i+1}\right)$ (каждой точки $t_{i}$ ) должно быть указано, совпадают данные ветви на данном интервале (в данной точке) или нет;

- непротиворечивость: о двух ветвях не может быть сказано, что они на данном интервале (в данной точке) совпадают и не совпадают одновременно;

- непрерывность: если две ветви совпадают на интервале $\left(t_{i}, t_{i+1}\right)$, то они совпадают и в точках $t_{i}, t_{i+1}$;

- симметричность: на данном интервале (в данной точке) если ветвь с номером $j$ совпадает (не совпадает) с ветвью с номером $k$, то и ветвь с номером $k$ совпадает (не совпадает) с ветвью с номером $j$;

- транзитивность: если на данном интервале (в данной точке) ветвь с номером $j$ совпадает с ветвью с номером $k$, а ветвь с номером $k$ совпадает с ветвью с номером $l$, то ветвь с номером $j$ совпадает с ветвью с номером $l$ на этом интервале (в этой точке).

Граф, полученный путем склеивания $m$ экземпляров отрезка $\left[t_{0}, t_{m}\right]$ согласно схеме, будем называть максимальным графом данной абстрактной схемы.

Теорема 2. Пусть задана абстрактная схема ветвления для $m$ ветвей $(T, m, A)$. Тогда в $\mathbb{R}^{n}$ при $n \geqslant 3$ существует хотя бы одна ветвящаяся моментная кривая с $m$ ветвями, представляющая собой вложение максимального графа данной абстрактной схемы.

Будем строить не просто ветвящуюся моментную кривую, а "выровненную" кривую - такую ветвящуюся моментную кривую, что ее ветви $S_{i}(t)=\vec{C}_{i}+$ $\int_{0}^{t} \vec{v}(\tau) k_{i}(\tau) d \tau$ удовлетворяют условиям: для всех $t_{j} \in T$ вектор-функции $S_{1}\left(t_{j}\right), S_{2}\left(t_{j}\right), \ldots, S_{m}\left(t_{j}\right)$ имеют одинаковую первую координату и функции $k_{i}(\tau)$ обращаются в нуль во всех точках $t_{j}$. Для доказательства потребуется

Лемма 1. Пусть задана “выровненная" ветвящаяся моментная кривая с $m$ ветвями

$$
S_{i}(t)=\vec{C}_{i}+\int_{0}^{t} \vec{v}(\tau) k_{i}(\tau) d \tau,
$$

из которых ветвъ с номером р выделена. Пусть для $t \leqslant t_{j}$ построена $(m+1)$-я ветвъ

$$
S_{m+1}=\vec{C}_{m+1}+\int_{0}^{t} \vec{v}(\tau) k_{m+1}(\tau) d \tau .
$$

Пусть $\vec{w}$ - некоторый вектор, перпендикулярный оси $x_{1}$.

Тогда существуют такие функции $\widehat{k}_{i}, i=1, \ldots, m+1$, что при замене $k_{i}$ на $\widehat{k_{i}}$ структура ветвления первых $m$ ветвей на всей оси $u(m+1)$-й ветви при $t<t_{j}$ не изменится, а ветвъ $S_{m+1}$ на отрезке $\left[t_{j}, t_{j+1}\right]$ будет вести себя таким образом, что $S_{m+1}\left(t_{j+1}\right)-S_{p}\left(t_{j+1}\right)=\vec{w}$.

ДокАЗАТЕльство. Заметим, что добавление любой бесконечно гладкой неотрицательной функции ко всем $k_{1}, k_{2}, \ldots, k_{m}$ не меняет структуру ветвящейся моментной кривой из $m$ ветвей. 
Рассмотрим случай $\vec{w} \neq S_{m+1}\left(t_{j}\right)-S_{p}\left(t_{j}\right)$. Зададим набор $n$ неотрицательных бесконечно гладких функций $p_{1}, p_{2}, \ldots, p_{n}$ с единичной интегральной мерой, носители которых непусты и содержатся в $\varepsilon$-окрестностях точек $\tau_{1}, \tau_{2}, \ldots, \tau_{n}, \tau_{i} \in\left(t_{j}, t_{j+1}\right)$, где $\varepsilon$ выбрано таким образом, чтобы $\varepsilon$-окрестности точек не пересекались и содержались в интервале $\left(t_{j}, t_{j+1}\right)$. Обозначим их добавочные векторы через $\vec{u}_{1}, \vec{u}_{2}, \ldots, \vec{u}_{n}$ соответственно. Так как при уменьшении $\varepsilon$ векторы $\vec{u}_{i}$ становятся сколь угодно близки к $\vec{v}\left(\tau_{1}\right), \vec{v}\left(\tau_{2}\right), \ldots, \vec{v}\left(\tau_{n}\right)$ - касательным векторам к обычной (не обобщенной) моментной кривой в точках $\tau_{i}$, а эти векторы линейно независимы по предложению 3 , существует $\varepsilon$ такое, что векторы $u_{i}$ будут линейно независимы. Первая координата всех этих векторов положительна по построению, так как первая координата вектора $\vec{v}$ равна единице, а функции $p_{i}$ неотрицательны и не равны тождественно нулю (потому что функции $p_{i}$ линейно независимы).

Разложим вектор $\vec{w}-S_{m+1}\left(t_{j}\right)+S_{p}\left(t_{j}\right)$ по базису $u: \alpha_{1} \vec{u}_{1}+\alpha_{2} \vec{u}_{2}+\cdots+\alpha_{n} \vec{u}_{n}=$ $\vec{w}-S_{m+1}\left(t_{j}\right)+S_{p}\left(t_{j}\right)$. Среди чисел $\alpha_{i}$ должны встречаться и положительные, и отрицательные, так как первая координата векторов $\vec{u}_{i}$ положительна, первая координата вектора $\vec{w}$ равна нулю, а у точек $S_{m+1}\left(t_{j}\right)$ и $S_{p}\left(t_{j}\right)$ первые координаты равны. Перенеся в правую часть слагаемые с отрицательными коэффициентами, получим

$$
\sum_{\alpha_{j}>0} \alpha_{j} \vec{u}_{j}=\vec{w}-S_{m+1}\left(t_{j}\right)+S_{p}\left(t_{j}\right)+\sum_{\alpha_{j}<0} \alpha_{j} \vec{u}_{j} .
$$

Сконструируем функции $\widehat{k}_{m+1}$ и все остальные $\widehat{k}_{i}$ следующим образом:

$$
\widehat{k}_{m+1}=k_{p}+\sum_{\alpha_{j}>0} \alpha_{j} p_{j}, \quad \widehat{k}_{i}=k_{i}-\sum_{\alpha_{j}<0} \alpha_{j} p_{j}, \quad i=1, \ldots, m
$$

(в частности, $\left.\widehat{k}_{p}=k_{p}-\sum_{\alpha_{j}<0} \alpha_{j} p_{j}\right)$. Функции $\sum_{\alpha_{j}>0} \alpha_{j} p_{j}$ и $-\sum_{\alpha_{j}<0} \alpha_{j} p_{j}$ неотрицательны, бесконечно гладки и их носители содержатся в отрезке $\left[t_{j}, t_{j+1}\right]$. Добавочными векторами для функций $\widehat{k}_{m+1}$ и $\widehat{k}_{p}$ служат, соответственно, векторы $\vec{r}_{1}=\vec{r}+\sum_{\alpha_{j}>0} \alpha_{j} \vec{u}_{j}$ и $\vec{r}_{2}=\vec{r}-\sum_{\alpha_{j}<0} \alpha_{j} \vec{u}_{j}$, где $\vec{r}-$ добавочный вектор функции $k_{p}$. Из построения очевидно, что эти векторы отличаются на $\vec{w}-$ $S_{m+1}\left(t_{j}\right)+S_{p}\left(t_{j}\right)$, а это в свою очередь означает, что $S_{m+1}\left(t_{j+1}\right)-S_{p}\left(t_{j+1}\right)=\vec{w}$. При $t_{j} \leqslant t \leqslant t_{j+1}$ кривая $S_{m+1}$ в общем случае не совпадает с другими, так как функции $p_{i}$ линейно независимы и имеется большой выбор таких функций. Случай разобран.

Теперь пусть $\vec{w}=S_{m+1}\left(t_{j}\right)-S_{p}\left(t_{j}\right)$. Зададим набор $l(l>n)$ неотрицательных бесконечно гладких функций $p_{1}, p_{2}, \ldots, p_{l}$, линейно независимых между собой, носитель каждой из которых непуст и содержится в отрезке $\left[t_{j}, t_{j+1}\right]$. Обозначим их добавочные векторы через $\vec{u}_{1}, \vec{u}_{2}, \ldots, \vec{u}_{l}$ соответственно. Так как $l>n$, система векторов $\vec{u}_{i}$ является линейно зависимой. Первая координата всех этих векторов положительна по построению, так как первая координата вектора $\vec{v}$ равна единице, а функции $p_{i}$ неотрицательны и не равны тождественно нулю (потому что функции $p_{i}$ линейно независимы). Запишем линейную комбинацию векторов $\vec{u}_{i}$, равную нулю: $\alpha_{1} \vec{u}_{1}+\alpha_{2} \vec{u}_{2}+\cdots+\alpha_{l} \vec{u}_{l}=0$. Среди 
чисел $\alpha_{i}$ должны встречаться и положительные, и отрицательные, так как первая координата векторов $\vec{u}_{i}$ положительна. Сконструируем функции $\widehat{k}_{m+1}$ и все остальные $\widehat{k}_{i}$ следующим образом: $\widehat{k}_{m+1}=k_{p}+\sum_{\alpha_{i}>0} \alpha_{i} p_{i}, \widehat{k}_{i}=k_{i}-\sum_{\alpha_{i}<0} \alpha_{i} p_{i}$, $i=1, \ldots, m$. Эти функции неотрицательны, бесконечно гладки и их носители содержатся в отрезке $\left[t_{j}, t_{j+1}\right]$. Добавочными векторами для функций $\widehat{k}_{m+1}$ и $\widehat{k}_{p}$ служат, соответственно, векторы $\vec{r}_{1}=\vec{r}+\sum_{\alpha_{i}>0} \alpha_{i} \vec{u}_{i}$ и $\vec{r}_{2}=\vec{r}-\sum_{\alpha_{i}<0} \alpha_{i} \vec{u}_{i}$, где $\vec{r}$ - добавочный вектор функции $k_{p}$. Из построения очевидно, что эти векторы равны. Это означает в свою очередь, что $S_{m+1}\left(t_{j}\right)-S_{p}\left(t_{j}\right)=S_{m+1}\left(t_{j+1}\right)-$ $S_{p}\left(t_{j+1}\right)=\vec{w}$. При $t_{j} \leqslant t \leqslant t_{j+1}$ кривая $S_{m+1}$ в общем случае не совпадает с другими, так как функции $p_{i}$ линейно независимы и имеется большой выбор таких функций. Случай разобран. Лемма доказана.

Заметим, что в условиях леммы, если кривая была “выровненной”, после продления $(m+1)$-й ветви она останется "выровненной”.

\section{4. Доказательство теоремы о реализации абстрактной схемы.}

ДокАзАтельство. Напомним, что мы будем доказывать больше, чем утверждается в теореме, а именно: существует подходящая "выровненная" моментная кривая такая, что ее ветви $S_{i}(t)=\vec{C}_{i}+\int_{t_{0}}^{t} \vec{v}(\tau) k_{i}(\tau) d \tau$ удовлетворяют условию: все функции $k_{i}$ обращаются в нуль во всех точках $t_{j}$. Заметим, что так как мы строим “выровненную" кривую, у всех векторов $\vec{C}_{i}$ должна быть одинаковая первая координата.

Проведем индукцию по числу ветвей. Для случая одной ветви утверждение очевидно: в качестве ветви $S_{1}$ возьмем обобщенную моментную кривую вида $S_{1}(t)=\int_{t_{0}}^{t} \vec{v}(\tau) k_{1}(\tau) d \tau$, где функция $k_{1}$ бесконечно гладкая, неотрицательная, обращается в нуль во всех точках $t_{i}$ и не обращается в нуль больше нигде.

Пусть теперь теорема доказана для не более чем $m$ ветвей. Докажем для $m+1$ ветви.

Вначале поступим так: проигнорируем ветвь с номером $m+1$ и все касающиеся нее условия. Мы получили абстрактную схему ветвления для $m$ ветвей. Воспользуемся предположением индукции и построим ветвящуюся моментную кривую, удовлетворяющую оставшимся условиям. Пусть ее ветви $S_{1}(t), S_{2}(t), \ldots, S_{m}(t)$, каждая из которых имеет вид $S_{i}(t)=\vec{C}_{i}+\int_{t_{0}}^{t} \vec{v}(\tau) k_{i}(\tau) d \tau$. В процессе доказательства мы не только построим новую ветвь $S_{m+1}=\vec{C}_{m+1}+$ $\int_{t_{0}}^{t} \vec{v}(\tau) \widehat{k}_{m+1}(\tau) d \tau$, но и видоизменим старые, поэтому будем говорить о конструировании $\vec{C}_{m+1}$ и $\widehat{k}_{i}(t)$, где $i=1, \ldots, m+1$.

Будем строить $\overrightarrow{\widehat{C}}_{i}, \widehat{k}_{i}$ на отрезках $\left[t_{j}, t_{j+1}\right]$ начиная с $j=0$ и далее по порядку. Положим $\widehat{k}_{m+1}\left(t_{0}\right)=0$. Если $(m+1)$-я ветвь в точке $t_{0}$ согласно условиям должна совпадать с некоторой ветвью $S_{i}$, положим $\vec{C}_{m+1}=\vec{C}_{i}$, иначе в качестве $\vec{C}_{m+1}$ возьмем произвольный вектор, не равный ни одному из $\vec{C}_{1}, \ldots, \vec{C}_{m}$, с такой же первой координатой, как у остальных $\vec{C}_{i}$. 
Итак, пусть на отрезке $\left[t_{0}, t_{j}\right]$ функции $\widehat{k}_{i}$ построены. Построим их на отрезке $\left[t_{j}, t_{j+1}\right]$. Рассмотрим два случая: случай, когда, согласно условиям, ветвь с номером $m+1$ на отрезке $\left[t_{j}, t_{j+1}\right]$ совпадает с какой-либо ветвью, и случай, когда она не совпадает ни с одной.

Пусть на отрезке $\left[t_{j}, t_{j+1}\right]$ ветвь с номером $m+1$ совпадает с некоторой ветвью $S_{r}$. Тогда на этом отрезке положим $\widehat{k}_{m+1}=k_{r}$. Все остальные $\widehat{k}_{i}$ совпадают с соответствующими $k_{i}$.

Рассмотрим случай, когда на интервале $\left(t_{j}, t_{j+1}\right)$ и в точке $t_{j+1}$ ветвь с номером $m+1$ не совпадает с другими ветвями. Выберем в качестве $S_{p}$ произвольную ветвь, а в качестве $\vec{w}$ - любой вектор, не совпадающий ни с одним из векторов $S_{i}\left(t_{j+1}\right)-S_{p}\left(t_{j+1}\right)$. Применим лемму 1. Ветвь построена.

Рассмотрим случай, когда на отрезке $\left[t_{j}, t_{j+1}\right]$ ветвь с номером $m+1$ не совпадает с другими ветвями, а в точке $t_{j+1}$ совпадает хотя бы с одной ветвью. Пусть ее номер $r$. Возьмем произвольный номер $p<m+1$, положим $\vec{w}=$ $S_{p}\left(t_{j+1}\right)-S_{r}\left(t_{j+1}\right)$ и применим лемму 1 . Ветвь построена. Теорема доказана.

3.5. Число минимаксного сечения. Теперь определим комбинаторную характеристику графа, от которой зависит верхняя оценка.

Рассмотрим некоторую нумерацию вершин графа. Для данной нумерации вершин введем частичный порядок на ребрах: ребро, соединяющее вершины $i_{1}$ и $i_{2}$ "не больше" ребра между вершинами $j_{1}$ и $j_{2}$, когда выполняются одновременно четыре неравенства $i_{1} \leqslant j_{1}, i_{2} \leqslant j_{1}, i_{1} \leqslant j_{2}, i_{2} \leqslant j_{2}$. Каждой нумерации поставим в соответствие "число максимального сечения" - максимальное число попарно несравнимых ребер. Оптимальной нумераиией назовем такую нумерацию вершин графа, при которой “число максимального сечения” минимально. Если таких нумераций несколько, будем считать оптимальной одну из них. "Число максимального сечения" оптимальной нумерации назовем числом минимаксного сечения. Последовательность ребер в графе назовем "согласованной с нумерацией цепью", если каждое следующее (по порядку прохождения) ребро "не больше" предыдущего. Такая "цепь" может быть несвязна.

Числом ветвистости графа назовем такое минимальное число $l$, что граф можно полностью покрыть $l$ "цепями", согласованными с оптимальной нумерацией.

ПреДЛОЖЕНИЕ 5. Число минимаксного сечения графа равно его числу ветвистости.

Это утверждение является переформулировкой теоремы Дилуорса [9], утверждающей, что в частично упорядоченном множестве длина максимальной антицепи равна минимальному числу цепей, на которые это множество можно разбить.

Воспользуемся нумерацией вершин для вложения графа в пространство.

ЗАмЕчАниЕ 2. Всякая нумерация вершин и деление графа на согласованные с нумерацией цепи порождают абстрактную схему ветвления. При этом ветви будут являться расширением цепи и совпадать или не совпадать, когда 
соответственно совпадают или не совпадают соответствующие цепи. Если в цепи имеются "пропуски", то мы вольны выбрать совпадение или несовпадение произвольным образом. При этом количество ветвей будет равно количеству цепей и в случае оптимальной нумерации равно числу минимаксного сечения.

3.6. Верхняя оценка числа гиперпланарности графов. Теперь, когда подготовительная работа сделана, перейдем к основным результатам.

ТЕОРема 3. При $n>2$ любой граф можно вложить в $n$-мерное пространство так, что число гиперпланарности образа будет не более чем $\mathrm{nl}$, где $l$ число ветвистости.

ДокАЗАТЕЛЬство. Построим абстрактную схему ветвления, соответствующую оптимальной нумерации вершин. В соответствии с замечанием 2 такая схема существует. Число ветвей в этой схеме можно сделать равным $l$ в силу замечания 2. По теореме 2 существует ветвящаяся моментная кривая, реализующая эту схему. По предложению 4 число гиперпланарности образа при этом вложении не превосходит $n l$. Теорема доказана.

\section{§ 4. Нижняя оценка в общем случае}

4.1. Определения и формулировка результата. Для формулировки нижней оценки потребуется следующее свойство графов.

ОПРедЕлЕниЕ 5. Выберем на графе $G n$ точек, которые далее будем называть "выделенными". Назовем отображение графа $G$ в прямую корректным, если:

- все выделенные точки отобразились в начало координат;

- если образы концов ребра не совпали, ребро линейно отображается в отрезок, соединяющий образы концов;

- если образы концов ребра совпали, выделим на этом ребре дополнительную точку, называемую серединой. Середина обязана отобразиться в точку прямой, не совпадающую с образом ни одной из вершин. Половины ребра линейно отображаются в отрезок, соединяющий образ середины с образом концов.

Кратностъю одномерной проекции называется максимум по всем возможным выборам выделенных точек минимума по всем корректным отображениям графа на прямую максимума кратности покрытия точки. При подсчете максимума кратности покрытия каждая выделенная точка считается не за один образ, а за $\lceil i / 2\rceil$, где $i$ - число выходящих из нее ребер. Выделенная точка, являющаяся точкой ребра, считается вершиной степени 2.

Заметим, что, так как все точки одного ребра с топологической точки зрения равноценны, выбор выделенных точек осуществляется конечным числом способов. Все корректные отображения графа в прямую с заданным порядком следования образов вершин и середин ребер эквивалентны с точки зрения максимальной кратности покрытия. Следовательно, комбинаторно различных 
корректных отображений данного графа на прямую конечное число. Таким образом, кратность одномерной проекции данного графа алгоритмически может быть вычислена за конечное время.

ПРЕДЛОЖЕНИЕ 6. Пусть задан граф $G$ с $k$ вершинами, на котором выделены $n$ точек. Рассмотрим $k$ точек на прямой (не обязательно различных) $x_{1}, x_{2}, \ldots, x_{k}$ и установим однозначное соответствие между вершинами гра$\oint а$ и этими точками. Пусть е $e_{1}, e_{2}, \ldots, e_{r}$ - такие ребра графа, кониы которых отобразились в совпадающие точки. Отметим на прямой точки (различные, ни одна из которых не совпадает ни с одной из $\left.x_{i}\right) y_{1}, y_{2}, \ldots, y_{r}$. На каждом

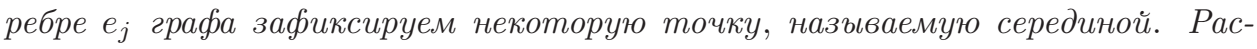
смотрим класс непрерывных отображсений графа на прямую таких, что:

- выделенные точки отобразились в начало координат;

- вериинь графа отобразились в соответствующие им точки $x_{i}$;

- для ребра е $e_{j}$, образы концов которого совпали, середина е в соответствуюиее $y_{j}$.

В классе таких отображений существует ровно одно корректное, и кратность покрытия каждой точки прямой при корректном отображении не выше, чем при любом другом отображении из этого класса.

Доказательство очевидно из принципа непрерывности.

ТЕОРемА 4. Кратность одномерной проекции оченивает число гиперпланарности снизу.

4.2. Леммы. Для доказательства теоремы потребуется следующая

Лемма 2. Пусть задано вложение $f$ графа $G$ в $n$-мерное пространство, задан набор из п различных выделенных точек образа графа $x_{1}, \ldots, x_{n}$. Пусть число гиперпланарности образа графа конечно. Пусть $d_{1}, d_{2}, \ldots, d_{n}-$ степени вершин $x_{1}, \ldots, x_{n}$ (точки ребра снова считаются вершинами второй степени). Тогда для любого $\varepsilon>0$ найдется гиперплоскость, отстоящая от каждой из отмеченных точек не более чем на $\varepsilon$, пересекающая в $\varepsilon$-окрестности каждой выделенной точки $x_{i}$ не менее чем $\left\lceil d_{i} / 2\right\rceil$ образов ребер.

ДоказАтельство. Сопоставим каждой вершине $x_{i}$ множество $B_{i} \subset S^{n-1}$ "плохих" направлений. Каждому вектору $w \in S^{n-1}$ соответствует ориентированная гиперплоскость, ортогональная $w$, проходящая через вершину $x_{i}$. Направление будем считать “плохим", если при малом сдвиге этой плоскости в направлении положительного вектора нормали гиперплоскость пересечет менее $\left\lceil d_{i} / 2\right\rceil$ образов ребер.

Заметим, что множества $B_{i}$ и $\sigma\left(B_{i}\right)$ на сфере не пересекаются. Действительно, если ни одно из ребер не лежит в гиперплоскости, ортогональной $w$ и проходящей через $x_{i}$, то в силу принципа Дирихле при малом сдвиге вдоль хотя бы одного из направлений $w$ и $-w$ гиперплоскость заденет не менее чем $\left\lceil d_{i} / 2\right\rceil$ образов ребер. Если одно из ребер частично принадлежит гиперплоскости, число гиперпланарности образа равно бесконечности, это противоречит условиям леммы. 
Докажем, что замыкания множеств $B_{i}$ и $\sigma\left(B_{i}\right)$ не пересекаются. Действительно, пусть существует вектор $\vec{u}$, принадлежащий как замыканию $B_{i}$, так и замыканию $\sigma\left(B_{i}\right)$. В любой окрестности вектора $\vec{u}$ найдутся как векторы из $B_{i}$, так и векторы из $\sigma\left(B_{i}\right)$. Это значит, что при сдвиге плоскости, ортогональной $\vec{u}$ и проходящей через $x_{i}$ как в направлении, сколь угодно близком к $\vec{u}$, так и в сколь угодно близком к противоположному, будет пересечено менее чем $\left\lceil d_{i} / 2\right\rceil$ образов ребер. Это возможно, только если хотя бы одно ребро частично лежит в плоскости, ортогональной $\vec{u}$ и проходящей через $x_{i}$. В таком случае число гиперпланарности бесконечно, это противоречит условиям леммы.

Так как замыкания множеств $B_{i}$ и $\sigma\left(B_{i}\right)$ не пересекаются, существует непрерывная функция $\varkappa_{i}: S^{n-1} \rightarrow[-1,1]$ такая, что $\left.\varkappa_{i}\right|_{B_{i}}=-1,\left.\varkappa_{i}\right|_{\sigma\left(B_{i}\right)}=1$. Можно потребовать, чтобы функции $\varkappa_{i}$ были кососимметричны.

Определим число $\delta$ следующим образом. В $\varepsilon$-окрестности каждой точки $x_{i}$ на каждом выходящем из нее ребре $e_{i, j}$ расположим $n$ точек таким образом, что вместе с $x_{i}$ они образуют симплекс. Если такие точки не найдутся, это означает, что часть ребра принадлежит гиперплоскости, случай тривиален. Пусть $\delta_{i, j}-$ ширина этого симплекса. Определим $\delta=\min \delta_{i, j} / 10$.

Для каждой точки $x_{i}$ и каждого вектора $w \in S^{n-1}$ определим "классическую гиперплоскость". Проведем через $x_{i}$ гиперплоскость, ортогональную $w$, и сдвинем ее в направлении $w$ на величину $\delta \varkappa_{i}(w)$. Заметим, что в силу выбора числа $\delta$ и в силу того, что $\left|\varkappa_{i}\right| \leqslant 1$ такой сдвиг достаточно мал для того, чтобы число пересеченных гиперплоскостью отрезков ребер, находящихся в $\varepsilon$-окрестности $x_{i}$, было больше чем $\left\lceil d_{i} / 2\right\rceil$, если направление $w$ не "плохое". Заметим также, что в силу кососимметричности функции $\varkappa_{i}$ "классическая гиперплоскость" для величин $w$ и $-w$ одна и та же, и она проходит через $x_{i}$, если $\varkappa_{i}(w)=0$. Если же $\varkappa_{i} \neq 0$, то "классическая" плоскость пересекает не менее чем $\left\lceil d_{i} / 2\right\rceil$ образов ребер.

Введем следующие функции $h_{i}: S^{n-1} \rightarrow \mathbb{R}, i=1, \ldots, n-1 ; h_{i}(w)$ равняется той величине, на которую нужно сдвинуть "классическую гиперплоскость" для точки $x_{i}$ и направления $w$, чтобы получить "классическую гиперплоскость" для точки $x_{n}$ и направления $w$. Так как при замене $w$ на $-w$ "классические гиперплоскости" останутся прежними, а положительное направление станет отрицательным, $h_{i}(-w)=-h_{i}(w)$.

Рассматривая $h_{i}$ как координатные функции, получим отображение $h=$ $\left(h_{1}, \ldots, h_{n-1}\right): S^{n-1} \rightarrow \mathbb{R}^{n-1}$. Воспользуемся теоремой Борсука-Улама. Существует пара противоположных направлений $w_{0}$ и $-w_{0}$ таких, что $h\left(w_{0}\right)=$ $h\left(-w_{0}\right)$. Заметим, что так как все функции $h_{i}$ антисимметричны, $h\left(w_{0}\right)=0$. Это означает, что "классическая гиперплоскость" для точки $x_{n}$ и направления $w_{0}$ является "классической" для всех $x_{i}$. Обозначим эту гиперплоскость через $\pi$.

Так как гиперплоскость $\pi$ является "классической гиперплоскостью" для всех $x_{i}$, для каждой из точек верно одно из двух: либо точка лежит на гиперплоскости $\pi$, либо в ее окрестности гиперплоскость $\pi$ пересекает большую часть исходящих ребер. Заметим, что если некоторая точка $x_{j}$ лежит в гиперплоскости $\pi$, это означает, что направления $w_{0}$ и $-w_{0}$ для $x_{j}$ не являются 
"плохими", и при сдвиге гиперплоскости в любом из этих направлений она пересечет не менее чем $\left\lceil d_{j} / 2\right\rceil$ образов ребер.

Если на плоскости $\pi$ лежат все точки $x_{i}$, сдвинем ее в направлении $w_{0}$ на $\delta$.

Рассмотрим случай, когда не все точки $x_{i}$ лежат в гиперплоскости $\pi$. Обозначим через $\varepsilon_{0}$ минимум по всем $x_{i}$, не лежащим на $\pi$, расстояния от $x_{i}$ до $\pi$. Сдвинем гиперплоскость $\pi$ в направлении $w_{0}$ на $\varepsilon_{0} / 2$. В окрестности точек, не принадлежащих $\pi$, число пересеченных ребер не изменится в силу выбора $\varepsilon_{0}$. В окрестности точек, принадлежащих $\pi$, новая гиперплоскость пересечет большую часть исходящих ребер в обоих случаях.

Итак, найдена гиперплоскость, пересекающая в $\varepsilon$-окрестности каждой вершины $x_{i}$ не менее чем $\left\lceil d_{i} / 2\right\rceil$ образов ребер. Лемма доказана.

Лемма 3. В условиях леммы 2 существует гиперплоскость, проходящая через выделенные точки, в сколь угодно малой окрестности которой существует гиперплоскость, пересекающая в в-окрестности каждой выделенной точки $x_{i}$ не менее чем $\left\lceil d_{i} / 2\right\rceil$ образов ребер.

Для доказательства достаточно из последовательности плоскостей из леммы 2 выделить сходящуюся подпоследовательность.

\section{3. Доказательство теоремы 4.}

ДокАЗАтЕЛьство. Пусть $G$ - произвольный, но фиксированный граф, число гиперпланарности которого $g$, а кратность одномерной проекции $k$. Рассмотрим такое вложение $f$ графа $G$ в $n$-мерное пространство, что число гиперпланарности образа равно $g$.

Обозначим через $s$ произвольный набор из $n$ точек графа, которые мы будем называть "отмеченными". Обозначим эти точки $x_{1}, x_{2}, \ldots, x_{n}$.

Применим к набору отмеченных точек лемму 3 . Обозначим через $\alpha$ плоскость, проходящую через отмеченные точки. Пусть $a$ - одномерная прямая, ортогональная $\alpha$. Пусть $p_{a}$ - проекция на прямую $a$ ортогонально плоскости $\alpha$. В соответствии с леммой 3 найдутся сколь угодно близкие к $\alpha$ гиперплоскости $\alpha_{j}$, пересекающие в окрестности каждой точки $x_{i}$ по меньшей мере $\left\lceil\operatorname{deg} x_{i} / 2\right\rceil$ ребер.

Обозначим через $m_{a}$ максимальную кратность покрытия на прямой $a$, причем, как и в определении кратности одномерной проекции, каждая "отмеченная" точка $x_{i}$ считается за $\left\lceil\operatorname{deg} x_{i} / 2\right\rceil$ образов (точка ребра снова считается вершиной степени два). Заметим, что $m_{a}$ равняется числу точек образа графа $G$ на достаточно близкой гиперплоскости $\alpha_{j}$. Так как число гиперпланарности множества $f(G)$ равно $g$, верно неравенство $m_{a} \leqslant g$.

Через $m_{s}$ обозначим минимум числа $m_{a}$ по всем направлениям $a$, перпендикулярным всем возможным гиперплоскостям, близким к образам "отмеченных" точек. Очевидно, $m_{s} \leqslant g$. Через $k_{s}$ обозначим минимум по всем корректным отображениям графа $G$ на прямую (для набора $s$ выделенных точек) максимума кратности покрытия точки, считая каждую отмеченную точку $x_{i}$ за $\left\lceil\operatorname{deg} x_{i} / 2\right\rceil$. По лемме $3 k_{s} \leqslant m_{s}$.

Теперь возьмем максимум по всем наборам $s$ как числа $k_{s}$, так и числа $m_{s}$. В первом случае мы получим $k$ - кратность одномерной проекции. Максимум 
числа $m_{s}$ обозначим через $m$. Получим $k \leqslant m$. Далее, так как $m_{s} \leqslant g$ и, следовательно, $m \leqslant g$, имеем $k \leqslant g$. Теорема доказана.

\section{§ 5. Верхняя оценка для деревьев}

\section{1. Формулировка результата.}

Теорема 5. Для любого дерева $G$ при $n \geqslant 3$ существует такое вложение в $n$-мерное пространство, что число гиперпланарности образа не превосходит $n+k-1$, где $k$ - минимальное число отрезков $A_{i}$, которыми можно покрыть дерево $G$ так, что любие два отрезка могут пересекаться только по вершине графа.

ЗАмЕчАниЕ 3. В этой теореме рассматривается "алгебраическое число пересечений" - каждая точка гиперплоскости считается столько раз, на каком числе отрезков она лежит.

Заметим, что число $k$ зависит исключительно от комбинаторных свойств графа. Ниже дано явное вычисление этого числа. Для доказательства теоремы нам потребуется следующее

ПреДЛОЖЕНИЕ 7. Пусть для двух непересекающихся отрезков на оси $t$ $\tau_{1}=\left[t_{1}, \widetilde{t}_{1}\right]$ u $\tau_{2}=\left[t_{2}, \widetilde{t}_{2}\right]$ выделены отрезки $\left.\left.l\right|_{\tau_{1}} u l\right|_{\tau_{2}}$ моментной кривой. Пусть к ним применены параллельные переносы $h_{1} u h_{2}$ соответственно, так что получены кривые $l_{1}=h_{1}\left(\left.l\right|_{\tau_{1}}\right)$ u $l_{2}=h_{2}\left(\left.l\right|_{\tau_{2}}\right)$. Пусть на кривой $l_{2}$ отмечена некоторая точка $A$, являющаяся общей точкой кривых. Тогда существует такой невырожденный отрезок $\widehat{\tau}_{2} \subseteq \tau_{2}$, что кривые $l_{1} u \widehat{l}_{2}=h_{2}\left(\left.l\right|_{\widehat{\tau}_{2}}\right)$ будут пересекаться только в точке $A$, причем свойство точки $A$ быть внутренней или граничной точкой кривой сохранится.

ДокАЗАТЕльство. По предложению 2 число точек пересечения кривых $l_{1}$ и $l_{2}$ конечно. Из этого факта очевидно следует существование подотрезка $\widehat{\tau}_{2}$, не содержащего других точек пересечения кроме $A$, такого, что для кривой $\widehat{l}_{2}$ сохранено свойство точки $A$ быть граничной либо внутренней точкой. Предложение доказано.

5.2. Доказательство теоремы 5. Рассмотрим классическую моментную кривую $l(t)=\left(t, t^{2}, \ldots, t^{n}\right)$. Заметим что каждый отрезок $\tau$ на оси $t$ порождает кривую $\left.l\right|_{\tau}$ в пространстве . Выберем на оси $t$ отрезок $\tau_{1}$. Зададим произвольный гомеоморфизм между $A_{1}$ и $l_{1}$.

Рассмотрим случай, когда один из отрезков $A_{i}$ пересекается с $A_{1}$. Без ограничения общности можем считать, что это $A_{2}$. Так как $G$ является деревом, точка пересечения $A_{1}$ и $A_{2}$ единственна. Выберем произвольный отрезок $\tau_{2}$ на оси $t$, не пересекающийся с $\tau_{1}$. Можно аналогичным образом задать соответствие между $l_{2}=\left.l\right|_{\tau_{2}}$ и $A_{2}$. Совместим образы вершины $A_{1} \cap A_{2}$, применив к $l_{2}$ параллельный перенос. Из предложения 7 вытекает, что выбором $\tau_{2}$ мы можем обеспечить отсутствие пересечения $l_{1}$ и $l_{2}$, за исключением образа вершины $A_{1} \cap A_{2}$. 
Рассмотрим случай, когда с $A_{1}$ не пересекается ни один из $A_{i}$. Выберем произвольный отрезок $\tau_{2}$ на оси $t$, не пересекающийся с $\tau_{1}$, и зададим соответствие между $l_{2}=\left.l\right|_{\tau_{2}}$ и $A_{2}$. Параллельно перенесем $l_{2}$ так, чтобы $l_{1}$ и $l_{2}$ не пересекались. Для обоих случаев построено вложение $A_{1} \cup A_{2}$ в пространство.

Аналогичным образом по индукции добавим к конструкции все остальные $l_{i}$ - образы $A_{i}$, сохраняя топологию их объединения на каждом шаге. Получим вложение дерева $G$ в пространство.

Докажем, что число гиперпланарности образа при полученном вложении не превышает $n+k-1$. Пусть некая гиперплоскость $\alpha$ пересекает образ графа по $r$ точкам. Пусть $\alpha$ пересекается с $l_{i}$ по $p_{i}$ точкам. Тогда если $p_{i}>1$ то из непрерывности следует существование $p_{i}-1$ касательных векторов $l_{i}$, параллельных $\alpha$. Если $p_{i} \leqslant 1$, число таких касательных векторов $\geqslant 0 \geqslant p_{i}-1$. Суммируя полученные неравенства по всем $i$, получаем, что число $q$ касательных векторов ко всем $l_{i}$, параллельных $\alpha$, удовлетворяет неравенству $q \geqslant \sum_{i}\left(p_{i}-1\right)=r-k$. По предложению $3 q \leqslant n-1$ (так как касательные векторы к $l_{i}$ равны касательным векторам к $l$ ). Из $r-k \leqslant q \leqslant n-1$ получаем $r \leqslant n+k-1$. Теорема доказана.

5.3. Асимптотические свойства оценок. Перейдем к доказательству асимптотических свойств последней полученной оценки. Применим к деревьям результат из предыдущего параграфа.

Теорема 6. Пусть задано дерево с вериинами $v_{1}, v_{2}, \ldots, v_{i}, \ldots$. Пусть размерность пространства не меньше числа вериин дерева степени 3 и въше. В таком случае кратность одномерной проекиии этого дерева не меньше следующей величинь:

$$
\sum_{\operatorname{deg} v_{i} \geqslant 3}\left(\left\lceil\frac{\operatorname{deg} v_{i}}{2}\right\rceil-1\right)+n .
$$

ДокАзАтельство. Пусть $V$ - количество вершин графа степени не ниже 3. Объявим все эти вершины “отмеченными" точками, а оставшиеся $n-V$ "отмеченных" точек разместим на ребрах графа произвольным образом.

Рассмотрим произвольное корректное отображение графа на прямую. Так как при подсчете кратности покрытия каждая отмеченная точка считается за $\lceil i / 2\rceil$, где $i$ - порядок точки, кратность покрытия образа выделенных точек (а значит, и максимальная кратность покрытия прямой) не менее величины $\sum_{\operatorname{deg} v_{i} \geqslant 3}\left\lceil\operatorname{deg} v_{i} / 2\right\rceil+n-V$. В силу произвольности корректного отображения, минимум по всем корректным отображениям максимума кратности покрытия также не менее этой величины. Кратность одномерной проекции также не менее этой величины, так как кратность одномерной проекции есть максимум минимума кратности покрытия.

Так как число $V$ можно представить как $\sum_{\operatorname{deg} v_{i} \geqslant 3} 1$, эту формулу можно переписать как

$$
\sum_{\operatorname{deg} v_{i} \geqslant 3}\left(\left\lceil\frac{\operatorname{deg} v_{i}}{2}\right\rceil-1\right)+n .
$$

Оценка кратности одномерной проекции получена. 
СледСтвиЕ 1. В условиях теоремы 6 число гиперпланарности дерева не меньше следующей величинь:

$$
\sum_{\operatorname{deg} v_{i} \geqslant 3}\left(\left\lceil\frac{\operatorname{deg} v_{i}}{2}\right\rceil-1\right)+n .
$$

Доказательство очевидно, так как кратность одномерной проекции оценивает снизу число гиперпланарности по теореме 4.

ТЕОрема 7. В условиях теоремы 6 (размерность пространства не меньше числа вершин дерева степени 3 и выше) верхняя оценка числа гиперпланарности для дерева, полученная в теореме 5, совпадает с нижней оценкой, полученной в следствии 1 и равна

$$
\sum_{\operatorname{deg} v_{i} \geqslant 3}\left(\left\lceil\frac{\operatorname{deg} v_{i}}{2}\right\rceil-1\right)+n .
$$

ДокАЗАтельство. Пусть $A_{1}, A_{2}, \ldots, A_{k}$ - минимальное покрытие дерева отрезками. Заметим, что эти отрезки могут пересекаться только в вершинах степени 3 и выше. Пересечение их в вершине степени 2 противоречит минимальности покрытия, так как в таком случае отрезки можно объединить. По аналогичной причине каждая вершина степени выше двух может быть концом не более чем одного отрезка $A_{i}$. Из этого следует, что в каждой вершине встречаются ровно $\left\lceil v_{i} / 2\right\rceil$ отрезков.

Эйлерова характеристика дерева равна 1. С другой стороны, дерево есть объединение $k$ отрезков $A_{i}$, эйлерова характеристика каждого из которых равна 1 , причем каждая вершина подсчитана столько раз, на каком числе отрезков она лежит. Так как каждая вершина лежит на $\left\lceil v_{i} / 2\right\rceil$ отрезков, получим формулу

$$
k-\sum_{\operatorname{deg} v_{i} \geqslant 3}\left(\left\lceil\frac{\operatorname{deg} v_{i}}{2}\right\rceil-1\right)=1 .
$$

Поменяем знак и добавим к каждой части равенства $n+k$, получим

$$
\sum_{\operatorname{deg} v_{i} \geqslant 3}\left(\left\lceil\frac{\operatorname{deg} v_{i}}{2}\right\rceil-1\right)+n=n+k-1 .
$$

Правая часть последнего равенства есть верхняя оценка числа гиперпланарности для деревьев из теоремы 5, а левая часть есть нижняя оценка из следствия 1. Теорема доказана.

Авторы благодарят своего научного руководителя С. А. Богатого за постановку задачи и помощь в работе.

\section{Список литературы}

[1] Т. А. Антонова, К. И. Облаков, "Специальные вложения графов в трехмерное пространство”, Вестн. Моск. ун-та. Сер. 1. Матем., мех., 2008, № 6, 26-31; англ. пер.: T. A. Antonova, K. I. Oblakov, "Special embeddings of graphs into a three-dimensional space", Moscow Univ. Math. Bull., 63:6 (2008), 245-250. 
[2] К. И. Облаков, Т. А. Облакова, "Специальные вложения некоторых несвязных графов в трехмерное пространство", Вестн. Моск. ун-та. Сер. 1. Матем., мех., 2011, № 2, 54-56; англ. пер.: Т. А. Oblakova, K. I. Oblakov, "Special embeddings of some disconnected graphs into Euclidean space", Moscow Univ. Math. Bull., 66:2 (2011), 90-92.

[3] K. Borsuk, "On the $k$-independent subsets of the Euclidean space and of the Hilbert space", Bull. Acad. Polon. Sci. Cl. III, 5:4 (1957), 351-356.

[4] В. Г. Болтянский, С. С. Рышков, Ю. А. Шашкин, "О $k$-регулярных вложениях и их применении к теории приближения функций”, УМН, 15:6 (1960), 125-132; англ. пер.: V. G. Boltyanskij, S.S. Ryshkov, Yu. A. Shashkin, "On k-regular imbeddings and their application to the theory of approximation of functions", Amer. Math. Soc. Transl. (2), 28 (1963), 211-219.

[5] С. А. Богатый, "Гипотеза Борсука, препятствие Рышкова, интерполяция, аппроксимация Чебышёва, трансверсальная теорема Тверберга, задачи", Дискретная геометрия и геометрия чисел, Тр. МИАН, 239, Наука, М., 2002, 63-82; англ. пер.: S. A. Bogatyĩ, "Borsuk's conjecture, Ryshkov obstruction, interpolation, Chebyshev approximation, transversal Tverberg's theorem, and problems", Proc. Steklov Inst. Math., 239 (2002), 55-73.

[6] С. А. Богатый, В. М. Вылов, "Вложения Робертса и обращение трансверсальной теоремы Тверберга", Матем. сб., 196:11 (2005), 33-52; англ. пер.: S. A. Bogatyi, V.M. Valov, "Roberts-type embeddings and conversion of transversal Tverberg's theorem", Sb. Math., 196:11 (2005), 1585-1603.

[7] J. C. Mairhuber, "On Haar's theorem concerning Chebychev approximation problems having unique solutions", Proc. Amer. Math. Soc., 7:4 (1956), 609-615.

[8] А. Брёнстед, Введение в теорию выпуклых многогранников, Мир, М., 1988; пер. с англ.: A. Brøndsted, An introduction to convex polytopes, Grad. Texts in Math., 90, Springer-Verlag, New York-Berlin, 1983.

[9] R. P. Dilworth, "A decomposition theorem for partially ordered sets", Ann. of Math. (2), 51:1 (1960), 161-166.

\section{К. И. Облаков (K. I. Oblakov)}

Механико-математический факультет

Московского государственного университета

им. М. В. Ломоносова

E-mail: v03oki@nm.ru

\section{T. А. Облакова (T. А. Oblakova)}

Механико-математический факультет

Московского государственного университета

им. М. В. Ломоносова

E-mail: oblakova.t@yandex.ru
Поступила в редакцию 14.04.2011 и 30.12 .2011 